\title{
Gastric Lipomatosis
}

\author{
In Ho Jeong, and Young Hee Maeng ${ }^{1}$ \\ Departments of Surgery and ${ }^{1}$ Pathology, Jeju National University School of Medicine, Jeju, Korea
}

Gastric lipomatosis is an extremely rare condition. We present a case of a 69-year-old woman admitted with epigastric soreness. Computerized tomography (CT) revealed extrinsically compressing, fat-containing mass lesions on the entire gastric wall of the antrum and body except for the lesser curvature. A subtotal gastrectomy was performed. Pathology findings confirmed a gastric lipomatosis with multiple gastric ulcerations and extensive disruptions of the muscular layers. This case and reports of other gastric lipomatosis cases indicate that CT should be used to characterize large submucosal masses because CT can show the specific nature and extent of the disease. We believe that surgical treatment is the most appropriate treatment for symptomatic gastric lipomatosis that shows extensive gastric involvement, or when there are multiple gastric lipomas.

Key Words: Stomach neoplasms, Lipomatosis, Gastrectomy

\section{Introduction}

Gastrointestinal lipoma is characterized by a round submucosal mass composed of well-differentiated adipose tissue surrounded by a fibrous capsule.(1,2) Gastrointestinal lipomatosis is commonly characterized by multiple gastrointestinal lipomas. However, a lipomatous lesion with diffuse infiltration on submucosal layer by adipose tissue is a distinct pathologic entity from conventional solitary lipoma. The previous reports suggested that the term "lipomatosis of intestine" more accurately describes the pathologic process and should replace the commonly used "lipoma of intestine". $(3,4)$ Therefore, gastric lipomatosis is characterized by multiple gastric lipomas or diffuse gastric infiltration on submucosal or subserosal layer by adipose tissue.

Gastrointestinal lipomas are one of the most common submucosal tumors in the gastrointestinal tract, and are usually small and asymptomatic. $(1,2)$ However, gastric lipomas are not common, and

\section{Correspondence to: In Ho Jeong}

Department of Surgery, Jeju National University School of Medicine, 102, Jeju Daehak-ro, Jeju 690-756, Korea

Tel: +82-64-723-2240, Fax: +82-64-726-0173

E-mail: 41056@naver.com

Received September 17, 2010

Accepted November 1, 2010 account for only $5 \%$ of gastrointestinal tract lipomas and fewer than $1 \sim 3 \%$ of all gastric tumors.(1,2) Gastric lipomatosis is an even rarer condition, with only ten previously reported cases.(3-10) Furthermore, only one report has described a gastric lipomatosis with extensive gastro-duodenal involvement that was not encapsulated.(8)

The present report describes the case of a patient who presented with epigastric soreness. Gastroscopy, barium studies and computerized tomography (CT) revealed diffuse fatty involvement in the stomach with ulceration. The patient was treated with a subtotal gastrectomy. Pathology examination of the resected specimen confirmed a gastric lipomatosis.

\section{Case Report}

A 69-year-old woman was admitted with intermittent epigastric soreness for the previous 3 months. She had no significant history of medical illness, did not drink alcohol, smoke or use NSAIDs. The family medical history was unremarkable. Laboratory tests returned results within normal limits, and included a tumor marker study, a complete blood count, urinalysis, serology, liver function studies, platelet count, coagulation time and clot retraction time. A review of the gastrointestinal system showed neither gastrointestinal bleeding nor obstructive symptoms. A physical examination of the 

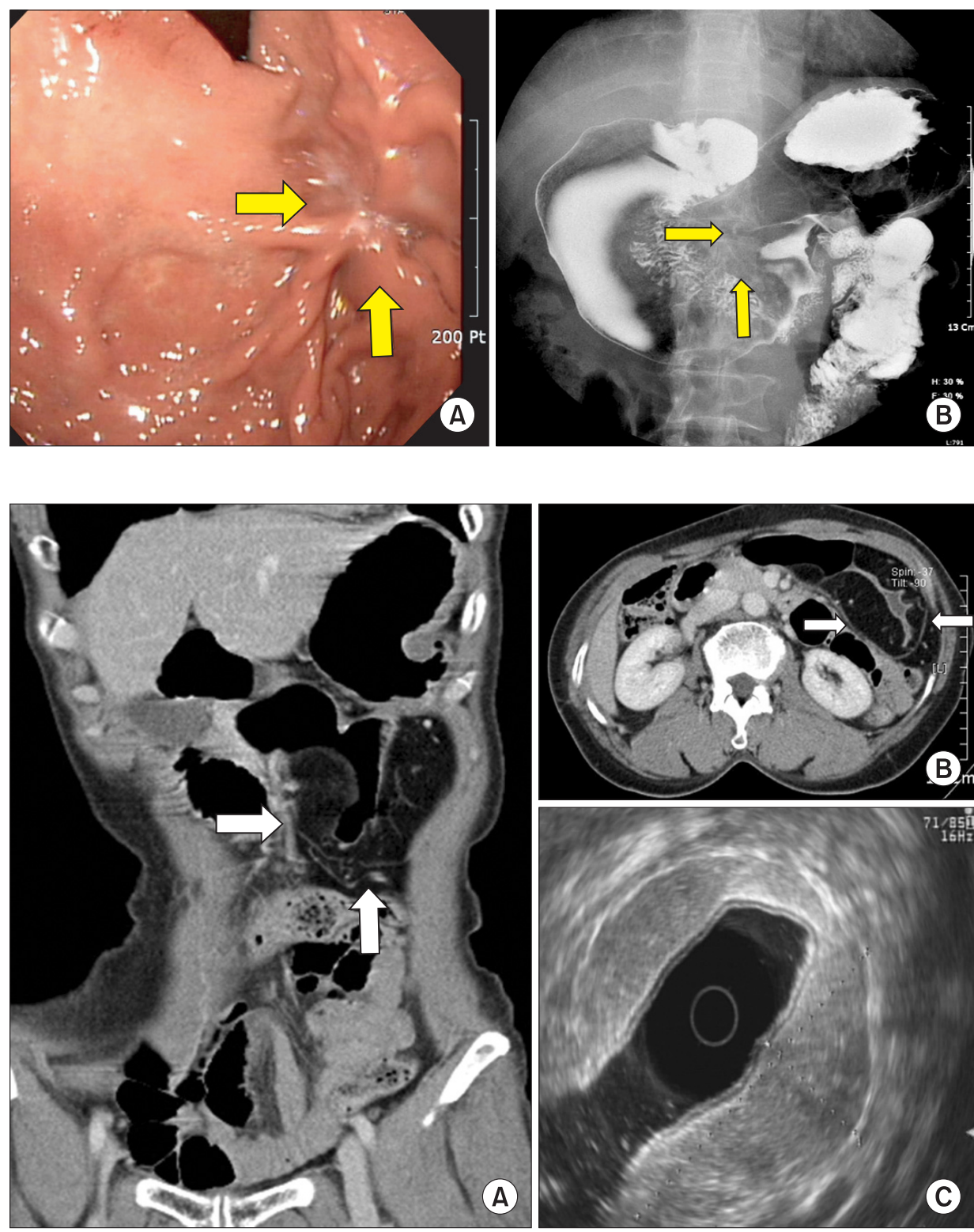

Fig. 1. Gastroscopy findings (A) and UGI study (B). Note the extrinsic compressing mass lesion on the anterior wall-greater curvature-posterior wall of the antrum and body, and the ulceration (arrows).
Fig. 2. CT (A and B) and EUS (C) radiology findings. Note the huge fatcontaining mass lesion around the gastric wall except for the lesser curvature, from the gastric midbody to the antrum. The lesions (arrows) appeared as well-circumscribed areas of fatty density with an attenuation ranging from -70 to $-120 \mathrm{H}$. The lesion originated at the submucosal layer, had a depth of about $2.7 \mathrm{~cm}$ and was homogeneously hyperechoic on EUS findings. abdomen indicated neither a palpable mass nor tenderness.

Gastroscopy and an upper gastrointestinal barium (UGI) study revealed an extrinsically compressing mass lesion on the anterior wall, greater curvature and posterior wall of the antrum and body of the stomach (Fig. 1). An abdominal CT showed an extrinsically compressing, large $(13 \times 8 \mathrm{~cm}$ maximum diameter $)$ fat-containing mass on the anterior wall, the greater curvature and the posterior wall from the gastric midbody to the antrum. This lesion appeared as well-circumscribed areas of fat density with an attenuation ranging from -70 to -120 Hounsfield units (Fig. 2A, B). There was no evidence of lymph node enlargement. Endoscopic ultrasonography (EUS) showed that the lesion originated at the submucosal layer, had a depth of approximately $2.7 \mathrm{~cm}$ and was homogeneously hy- perechoic (Fig. 2C).

The lipoma had multiple gastric ulcerations and extensive gastric involvement, which differentiated it from a conventional solitary lipoma. A diagnosis was made of a symptomatic huge gastric submucosal tumor. The patient underwent a subtotal gastrectomy with a Billroth II anastomosis.

Histological testing of the resected specimen confirmed the diagnosis of gastric lipomatosis. The gross pathological findings were a smooth glistening serosal surface with multiple nodules of deep yellowish adipose tissue without a fibrous capsule (Fig. 3). The lesion was not encapsulated nor was it well circumscribed, which suggested lipomatosis rather than a lipoma. The specimen was opened along the greater curvature. The mucosal surface was found 

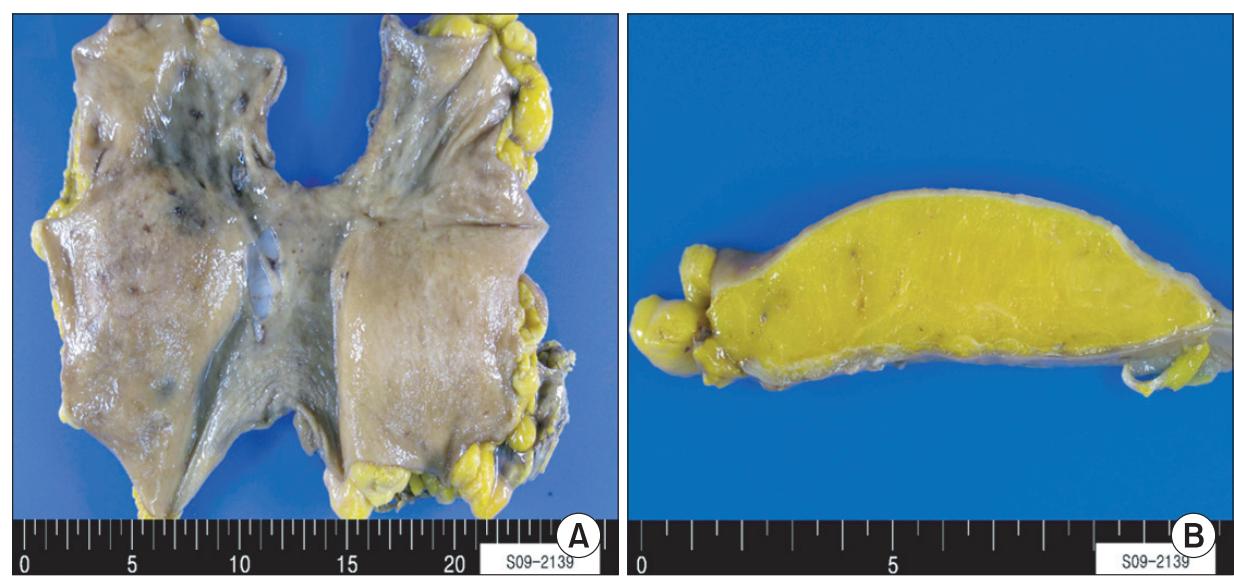

Fig. 3. Gross pathology findings of the resected specimen. (A) Examination of the intact specimen. Note the multiple nodules of deep adipose tissue without encapsulation, and the mucosal surface with several ulcers which was diffusely elevated by the submucosal mass (approximately $16 \times 16 \mathrm{~cm}$ ) that almost encircled the gastric wall. (B) Examination of the specimen following sectioning. Note the deep adipose tissue mainly in the submucosa, and adipose tissue diffusely disrupting the muscular wall.
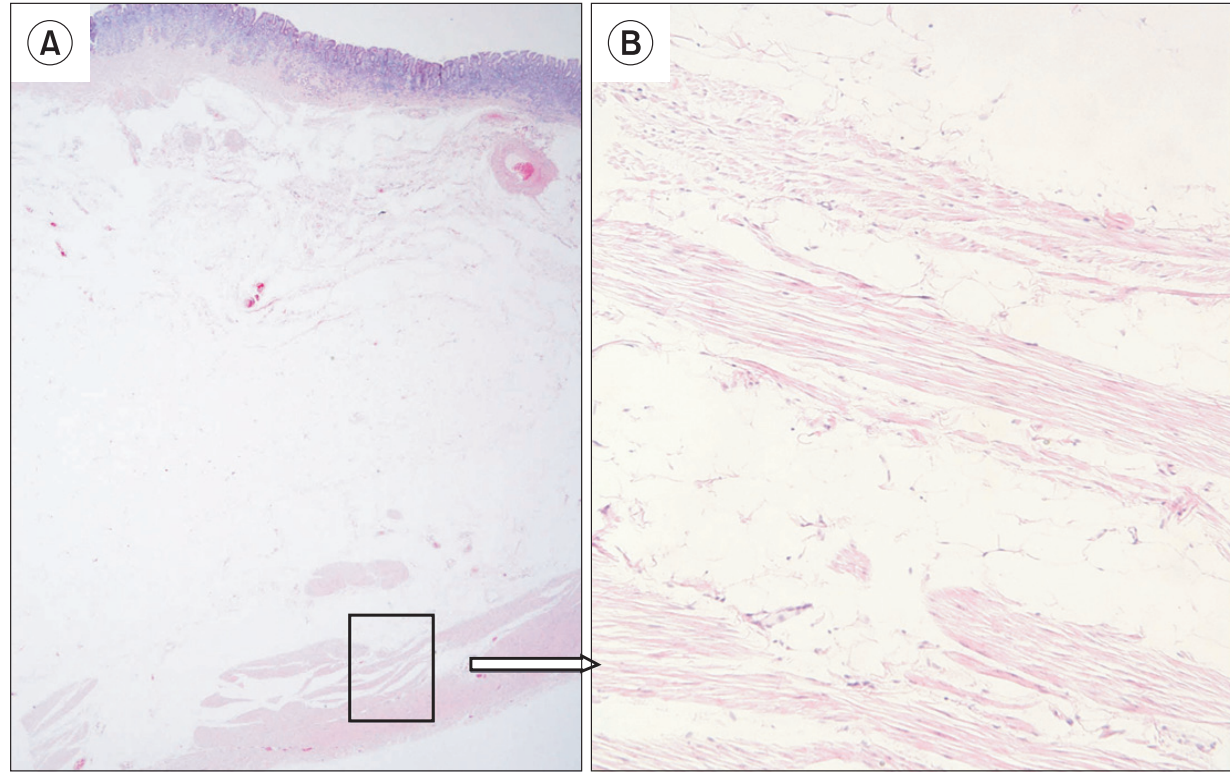

Fig. 4. Photomicrographic findings. Note the mature adipocytes replacing the submucosa and the muscle layer of the stomach $(\mathrm{A}: \mathrm{H} \& \mathrm{E}, \times 10$ and $\mathrm{B}$ : H\&E, $\times 200$ ) to have several ulcers and was diffusely elevated by the submucosal mass (approximately $16 \times 16 \mathrm{~cm}$ ), which almost encircled the gastric wall except for the lesser curvature. Sectioning revealed welldifferentiated deep yellowish adipose tissue mainly in the submucosa which diffusely disrupted the muscular wall. The proximal and distal resection margins were $1.5 \mathrm{~cm}$ and $2 \mathrm{~cm}$ distant from the adipose tissue mass, respectively. Photomicrographic findings showed infiltrative growth of mature adipocytes that replaced the submucosa and muscle layer of the stomach (Fig. 4). There was no evidence of malignancy in ulcers and a fatty lesion.

The patient recovered well after surgery and was discharged on postoperative day 10. The latest follow-up at postoperative 2 years found that the patient was continuing to do well, and had no evidence of recurrence.

\section{Discussion}

Gastric lipomas are characterized by a smooth, sharply marginated, oval or spherical submucosal mass composed of welldifferentiated adipose tissue surrounded by a fibrous capsule.(1,2,11) Gastric lipomatosis is characterized by multiple gastric lipomas or diffuse gastric infiltration on submucosal or subserosal layer by adipose tissue. $(3,4,11)$ The previous reports described diffuse infiltration on submucosal layer by adipose tissue as a pathologic process and suggested that the term "lipomatosis or lipohyperplasia of intestine" should be replace the term "lipoma of intestine". $(3,11)$ The etiology of gastric lipomatosis remains to be established. Postulated etiological factors include embryonic displacement of adipose tissue, congenital predisposition, degenerative disease with disturbance of fat metabolism, post-chemotherapeutic fat deposition, chronic irritation such as chronic inflammatory bowel disease, low-grade 
infection and hamartomatous syndromes. $(8,10,11)$ Cabaud and Harris(11) proposed that a fatty lesion with an annular nature and complete lack of encapsulation is a fatty infiltration rather than a true neoplasm. They described a pathological process whereby mechanical propulsion of the mucosa and submucosa downstream into the lumen of the bowel might increase the submucosal area which is then filled by fat. No report has described malignant transformation of gastric lipomas. $(5,12,13)$

Gastric lipomas are usually small, solitary and asymptomatic, and are hence often detected incidentally.(1,2) Hundreds of gastric lipoma cases have been reported in the medical literature. $(3,4) \mathrm{Al}-$ though lipomas are occasionally detected within the gastrointestinal tract and account for $4 \sim 8 \%$ of all gastrointestinal tract tumors, gastric lipomas are uncommon. According to a survey of gastric submucosal tumors that were surgically resected during a 2 year period in Korea, gastric lipomas accounted for $0.9 \%$ of all gastric submucosal tumors.(14)

Approximately $75 \%$ of gastric lipomas are located in the antrum. In addition, approximately 90 95\% of gastric lipomas are located in the submucosal layer, with the remainder in the subserosal layer.(1) Clinical symptoms are related to tumor size and location, and the development of complications. $(1,12)$ The most common clinical presentation $(50 \%)$ is reported to be upper gastrointestinal bleeding caused by ulceration of the tumor. $(1,12)$ Obstructive symptoms including vomiting and gastrointestinal obstruction are also frequent $(42 \%) .(1,12)$ The development of complications including gastrointestinal bleeding caused by ulceration and gastrointestinal obstruction can be manifested clinically when gastric lipomas are larger than $2 \mathrm{~cm} .(1,12)$

The medical literature contains few reports of gastric lipomatosis.(3-10) We found 9 case reports describing multiple gastric lipomas, and 1 case report describing diffuse depositions of adipose tissue that were not encapsulated. Eight of those 10 patients first presented due to upper gastrointestinal bleeding, and the other 2 patients had non-specific gastrointestinal symptoms. Although gastrointestinal bleeding generally presented as chronic blood loss, 2 patients had acute severe blood loss resulting in emergency presentations. $(4,5)$ Two of the 10 patients died due to massive gastrointestinal bleeding. The 9 case reports of multiple lipomas described encapsulated tumors.

To the best of our knowledge, the present report is the first to describe a patient with lipomatosis that showed extensive gastric involvement and required surgical removal because it was symptomatic. Although a previous report also described a case featuring extensive gastro-duodenal involvement, that patient was asymptomatic.(8) That patient was followed-up for 5 years and showed good recovery and no disease progression.

Abdominal CT is the imaging method of choice for determining the specific nature of a lipoma, the extent of the disease and for follow-up.(2,12) A homogeneous gastric submucosal tumor with an attenuation of between -70 and -120 Hounsfield units has been reported as a definitive finding for the diagnosis of gastric lipoma. $(2,12)$ Therefore, if a large submucosal tumor is detected on an endoscopic or UGI examination, then a CT scan should be obtained for making the diagnosis and deciding on the method of therapy.

Conservative treatment is preferred for solitary lipomas that are asymptomatic. However, further evaluation and surgical management should be planned when endoscopic examination shows an ulcer or when tumors contain non-fatty elements, are symptomatic or show infiltrative growth patterns. $(1,2,4,9,12)$ The current patient presented with epigastric soreness and non-specific gastrointestinal symptoms, but endoscopic examination ruled out the possibility of reflux disease or duodenal ulcer disease. A decision was made to operate due to the risk of bleeding associated with gastric ulcers.

In conclusion, CT should be used to characterize large submucosal masses because it can show the specific nature of a mass and the extent of disease. In addition, surgical treatment is appropriate in symptomatic patients with extensive gastric involvement.

\section{References}

1. Kim HS, Noh SH, Kim CK. Lipomas of gastrointestinal tract. J Korean Surg Soc 1989;36:98-108.

2. Ferrozzi F, Tognini G, Bova D, Pavone P. Lipomatous tumors of the stomach: CT findings and differential diagnosis. J Comput Assist Tomogr 2000;24:854-858.

3. Suárez Moreno RM, Hernández Ramírez DA, Madrazo Navarro M, Salazar Lozano CR, Martínez Gen R. Multiple intestinal lipomatosis. Case report. Cir Cir 2010;78:163-165.

4. Peabody JW Jr, Zikind J. Lipomatosis of the stomach; a case report and a review of the literature. Ann Surg 1953;138:784790.

5. Ventura L, Leocata P, Guadagni S, Ventura T. Multiple gastric lipomas: report of an asymptomatic case found at autopsy. Pathol Int 1997;47:575-577.

6. Skinner MS, Broadaway RK, Grossman P, Seckinger D. Multiple gastric lipomas. Dig Dis Sci 1983;28:1147-1149.

7. Deeths TM, Madden PN, Dodds WJ. Multiple lipomas of the 
stomach and duodenum. Am J Dig Dis 1975;20:771-774.

8. Devlies F, Hoe LV, Leemans A, Ponette E, Paepe ID. Gastroduodenal lipomatosis. Eur Radiol 1997;7:338-340.

9. Fawcett NW, Bolton VL, Geever EF. Multiple Lipomas of the Stomach and Duodenum. Ann Surg 1949;129:524-527.

10. Weinberg T, Feldman M. Lipomas of the gastrointestinal tract. Am J Clin Pathol 1955;25:272-281.

11. Cabaud PG, Harris LT. Lipomatosis of the ileocecal valve. Ann Surg 1959;150:1092-1098.
12. Thompson WM, Kende AI, Levy AD. Imaging characteristics of gastric lipomas in 16 adult and pediatric patients. AJR Am J Roentgenol 2003;181:981-985.

13. Siegal A, Witz M. Gastrointestinal lipoma and malignancies. J Surg Oncol 1991;47:170-174.

14. The Information Committee of the Korean Gastric Cancer Association. 2005-2006 nationwide gastric submucosal tumor report in Korea. J Korean Gastric Cancer Assoc 2008;8:104111. 